\title{
Why Chronic Constipation May be Harmful to Your Lungs: A Case Report and Review of Lipoid Pneumonia and Mycobacterium fortuitum Leading to Acute Respiratory Failure and Septic Shock
}

\author{
Sandra Till, DO \\ Manoj Mathew, MD \\ Da-Wei Liao, MD \\ Christina Ramirez, MD \\ Banner University Medical Center \\ Phoenix, AZ
}

\section{Case Report}

A 43 year-old female with a past medical history of right-sided hemiparesis secondary to motor vehicle accident 17 years prior presented a two week history of cough, fever and right-sided pleuritic chest pain. Her baseline status included using a wheelchair, living alone at home and working as a teacher.

On admission she had a temperature of $39.6^{\circ} \mathrm{C}$, was tachycardia and hypotensive requiring vasopressors. Labs were remarkable for a white count of 25,000 cells $/ \mathrm{mcL}$. Chest $\mathrm{x}$-ray showed right-sided infiltrate and pleural effusion (Figure 1).

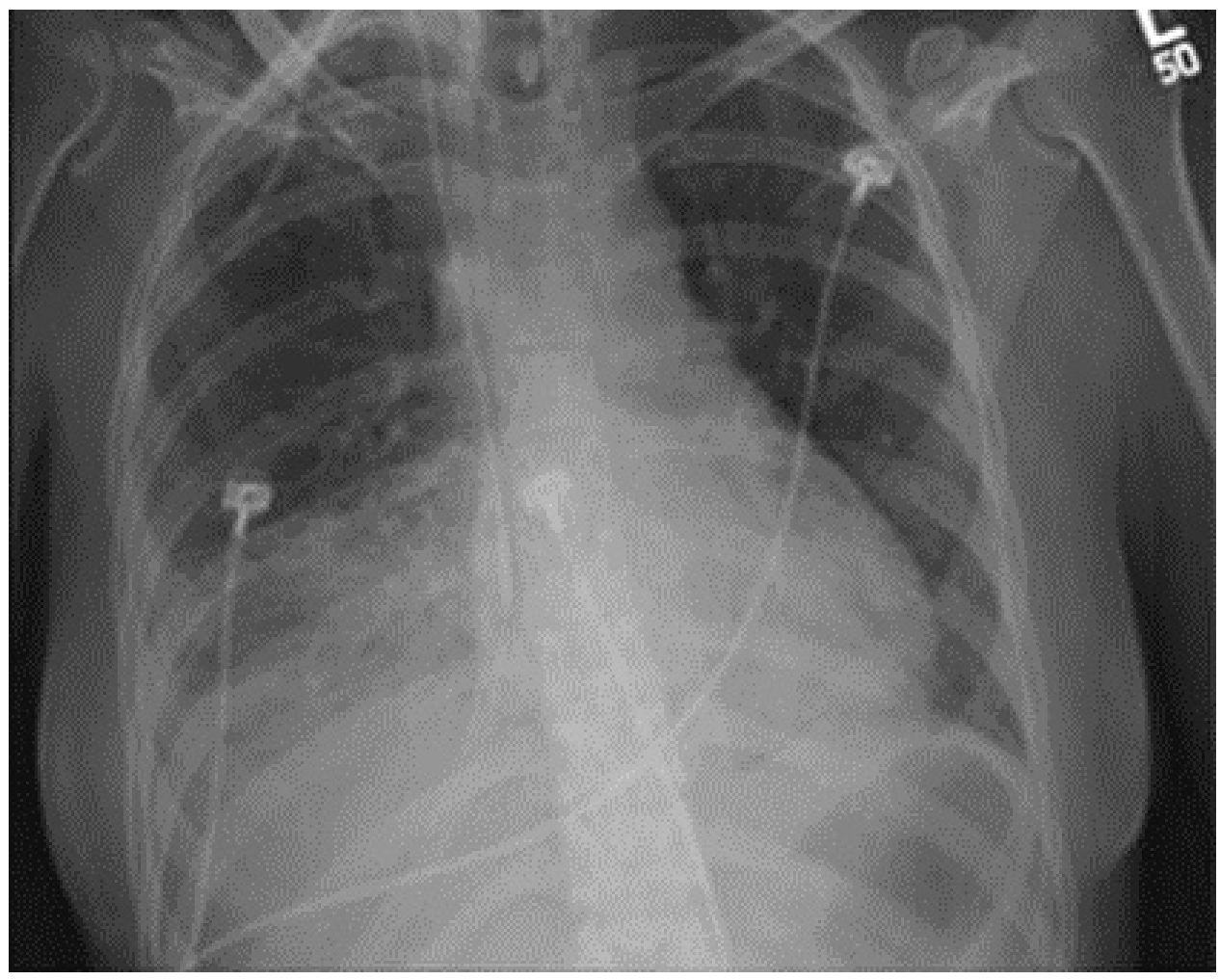

Figure 1. Chest x-ray on presentation. 
Bronchoscopy and thoracentesis was performed upon admission. The pleural fluid was exudative with a glucose of 78 and no suggestion of loculations on chest x-ray or ultrasound. The patient was started on therapy for community-acquired pneumonia.

On day 4 after admission, the patient had increasing sinus tachycardia, hypotension and was worsening despite being on antimicrobial therapy. A CT angiogram of the chest was performed (Figure 2).

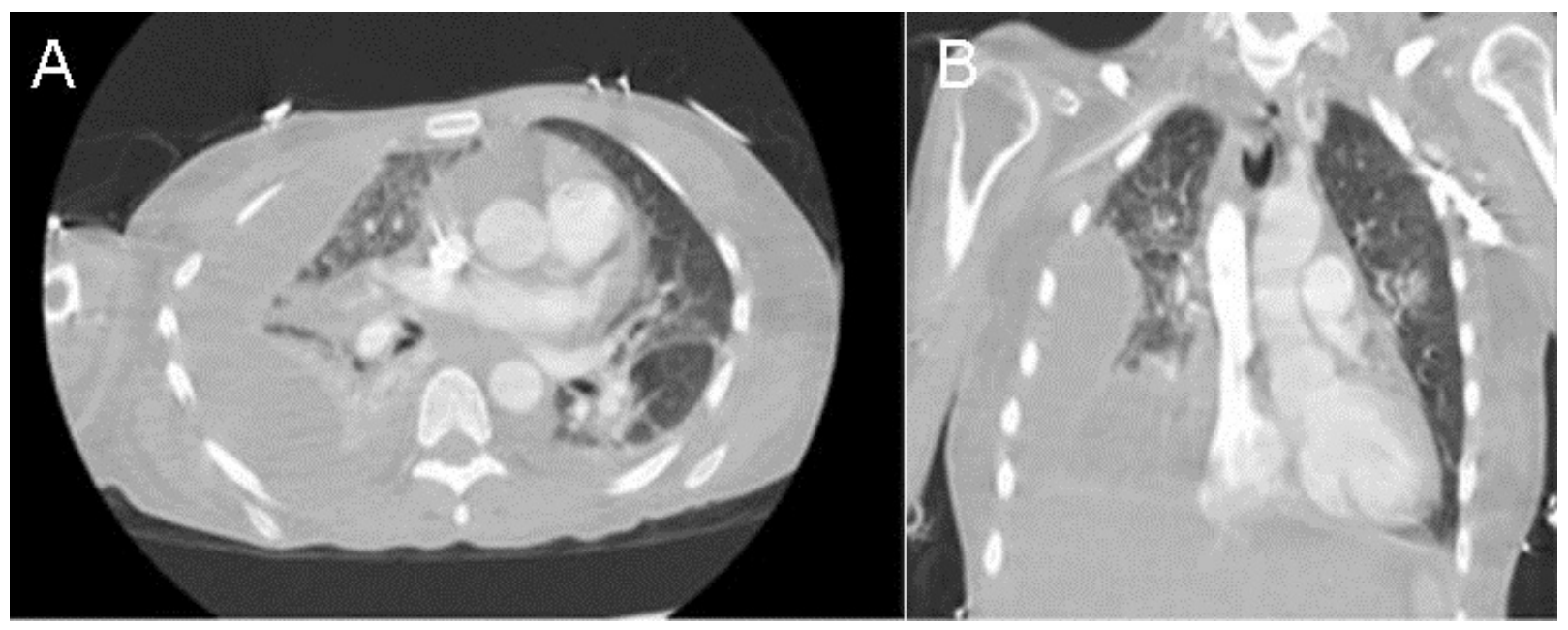

Figure 2. Initial CT scan on day 4 of admission. Panel A: axial view showing pneumonia and right pleural effusion. Panel B: coronal view.

CT angiogram was negative for pulmonary embolism and a percutaneous chest tube was placed on day 4 for drainage of pleural effusion due to development of loculations. On day 7 , the pleural fluid from initial thoracentesis grew acid-fast bacteria identified as Mycobacterium fortuitum.

Bronchoscopy was performed on day 8 and there was no endobronchial obstruction. Bronchoscopic alveolar lavage cultures grew Mycobacterium fortuitum. She had no history of bronchiectasis, skin infection, or immunoglobulin deficiency. Treatment with amikacin and levofloxacin was initiated based on susceptibilities.

The pleural chest tube was removed on day 14 (Figure 3). At this time the patient was transferred to a skilled nursing facility. 


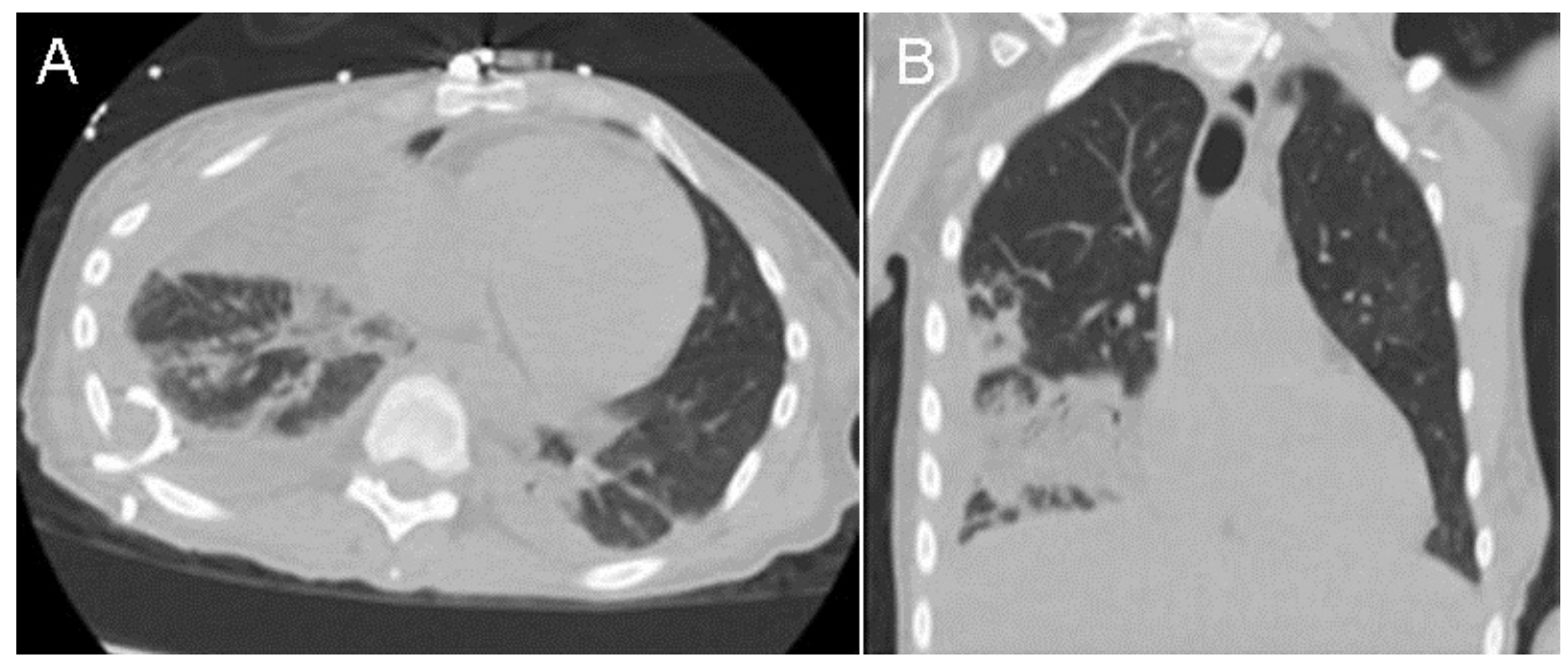

Figure 3. CT scan on day 13 prior to chest tube removal. Panel A: axial view. Panel B: coronal view.

The patient continued antibiotic treatment for Mycobacterium fortuitum with amikacin and levofloxacin, however, serial sputum cultures remained positive. On day 25, in the skilled nursing facility, the patient developed respiratory failure due to increased right effusion and worsening pneumonia. She was transferred to our facility were she was intubated and a new right-sided chest tube was placed. After placement of chest tube and drainage the right lung did not expand. Decompensation was felt to be related to the inadequate evacuation of the empyema with plans to solely continue antimicrobial therapies by the outside facility.
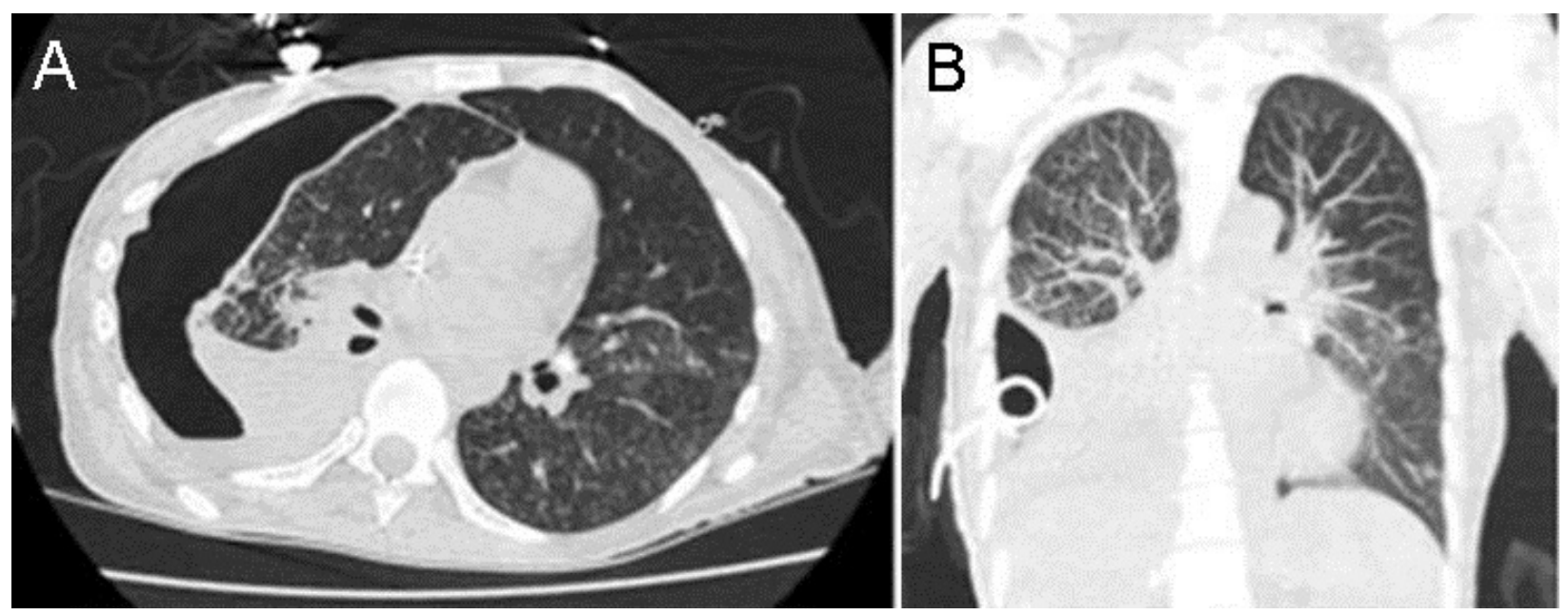

Figure 4. CT scan on day 30 showing trapped lung. Panel A: axial view. Panel B: coronal view.

Repeat pleural fluid cultures and BAL once again grew Mycobacterium fortuitum. She was taken for decortication and right middle and lower lobe resection by thoracic 
surgery. Due to extensive disease the patient required right thoracotomy, decortication, parietal pleurectomy, right middle lobectomy, and wedge resection of a right lower lobe lung abscess.

The lung pathology is shown below and was consistent with lipoid pneumonia (Figure $5)$.

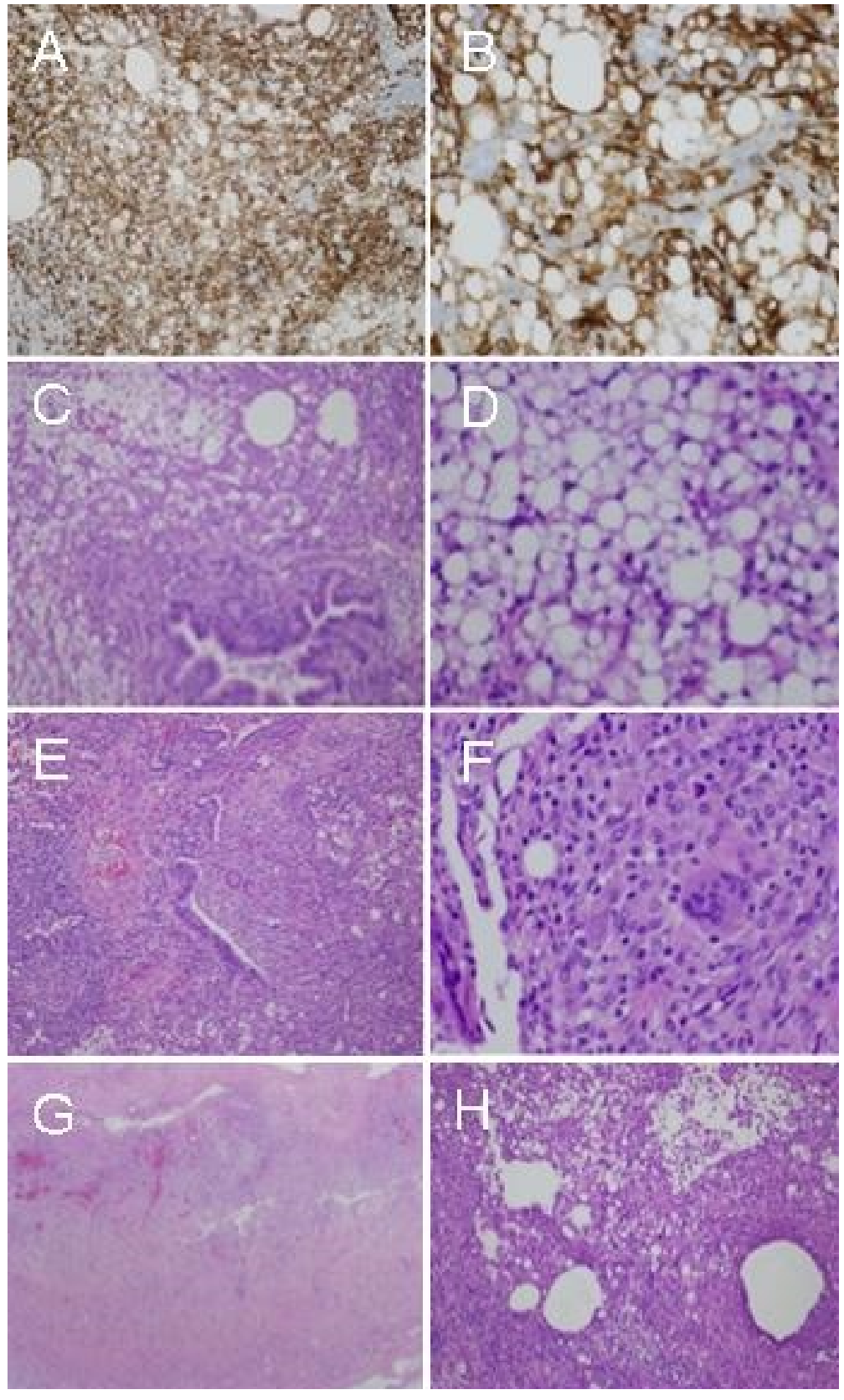

Figure 5. Panels A \& B: CD 163 stains showing lipid present within histiocytes. Panels C \& D: histology demonstrating severe lipoid pneumonia. Panels E \& F: Granulomatous inflammation with giant cells. Panel G: pleura. Panel H: abscess. 
There were no mycobacteria cultured on the lung biopsy. There were areas of both acute and chronic fibrosis noted on pathology report along with areas of acute interstitial pneumonitis and granulomatous inflammation.

During post-operative phase the patient confirmed that she was drinking mineral oil chronically for treatment of constipation. Repeat sputum cultures 7 days post operatively were negative for Mycobacterium fortuitum. She continued to improve with treatment of Mycobacterium fortuitum and postoperative cultures remained negative. She was able to liberate from the ventilator and returned home at after a prolonged course of rehabilitation.

\section{Lipoid Pneumonia and Associated Mycobacterial Infection}

The association between acid-fast bacteria and lipoid pneumonia was first reported in 1925 and since case reports have been noted. In 1953, a case report and literature review documented six cases of "saprophytic" mycobacteria was noted in conjunction with lipoid pneumonia. It was observed at this time that the fatty environment of lipoid pneumonia might assist with the growth of mycobacterium (1). Since then, intermittent case reports have been published reporting lipoid pneumonia with atypical mycobacteria.

There are two main categories of lipoid pneumonia, endogenous and exogenous. The endogenous form is also known as cholesterol pneumonia or golden pneumonia. It is associated with lysis of lung tissue distal to obstruction due to malignancy, fat storage disease such as Neiman-Pick or Gaucher's, medications and therapies including chemotherapeutic agents, amiodarone and radiation therapy. Pulmonary alveolar proteinosis has also been reported in idiopathic cases with granulomatosis with polyangiitis and connective tissue diseases (2-4). In polarized light microscopy after staining with sulfuric and acetic acid, the sample reveals cholesterol crystals, which is diagnostic of endogenous lipoid pneumonia (3).

Exogenous lipoid pneumonia occurs when external substances enter the lungs due to inhalation or aspiration (3). Cases have been reported from mineral oil, paraffin use, oil based nasal drops, total parenteral nutrition, mineral oil nose drops, black fat tobacco smoking, milk, and liquid hydrocarbons used by flame blowers (2-6). The pulmonary reaction to each substance varies. For example, mineral oils are fairly inert and less likely to produce alveolar inflammation, where milk fats are hydrolyzed by lung lipases leading to a significant inflammatory response (2).

The clinical presentation and appearance of lipoid pneumonia is variable from consolidation to effusion to nodule. Nodules from lipids may have elevated standardized uptake value (SUV) on positron emission tomography (PET) scan. The BAL from lipoid pneumonia may demonstrate lipid laden foamy macrophages (2). Mineral oil granuloma (paraffinoma) also can present as a spiculated mass mimicking malignancy. 
Mineral oil is notorious for causing lipoid pneumonia by aspiration for several reasons. First, it floats on the column of undigested material in the esophagus so it is first to be aspirated (5); secondly, it impairs phagocytosis at the alveolar level; and lastly, it inhibits the cough reflex and motor function of ciliated mucosa (7).

The impairment of phagocytosis associated with lipoid pneumonia is thought to be a contributing factor in why atypical mycobacterium strives in the lipid rich environment of lipoid pneumonia $(5,6)$. Malnutrition is also thought to be a component of risk as it due to impairment in cell mediated immunity (6). Lipid acts as mechanical protection for the mycobacteria favoring tissue necrosis facilitating secondary infection. Also it is thought that lipids may activate the cell walls of the atypical mycobacteria leading to increased virulence of the mycolic acids within the wall of the bacteria (8).

Mycobacterium fortuitum rarely causes pulmonary disease unless associated with lipoid pneumonia. This is often related to gastroesophageal disease and chronic vomiting and aspiration of contents. It is typically associated with skin and soft tissue infections and is a rapid growing mycobacterium and most frequently found in water and soil $(2,8,9)$

This case demonstrates an atypical presentation of lipoid pneumonia and Mycobacterium fortuitum infection leading to septic shock and ventilator failure. Although the association of lipoid pneumonia and mycobacterial infections is well documented, the rapid and acute decline in this patient's clinical status is unusual. This can be attributed to incomplete drainage of the initial empyema prior to transfer to the skilled nursing facility.

The etiology of the lipoid pneumonia was chronic aspiration of mineral oil producing an ideal environment for growth of Mycobacterium fortuitum. The absence of bronchiectasis, immunoglobin deficiency, skin infections should prompt further evaluation for abnormal lung architecture serving as a nidus for Mycobacterium fortuitum Infection. In our case, failure to improve is attributed to a persistent nidus for infection. We advocate resection of diseased lung segments of lipoid pneumonia to facilitate successful treatment of Mycobacterium fortuitum. In conclusion, if a patient has lipoid pneumonia with signs of clinical infection, the possibility of rapidly growing mycobacterium such as $\mathrm{M}$. fortuitum should be considered.

\section{References}

1. Gibson JB. Infection of the lungs by saprophytic mycobacteria in achalasia of the cardia, with report of a fatal case showing lipoid pneumonia due to milk. J Pathol Bacteriol. 1953;65(1):239-51. [CrossRef] [PubMed]

2. Hasan A, Swamy T. Nocardia and Mycobacterium fortuitum infection in a case of lipoid pneumonia. Respiratory Medicine CME 2011: 75-78. [CrossRef]

3. Betancourt SL, Martinez-Jimenez S, Rossi SE, Truong MT, Carrillo J, Erasmus JJ. Lipoid pneumonia: spectrum of clinical and radiologic manifestations. AJR Am J Roentgenol. 2010;194(1):103-9. [CrossRef] [PubMed] 
4. Harris K, Chalhoub M, Maroun R, Abi-Fadel F, Zhao F. Lipoid pneumonia: a challenging diagnosis. Heart Lung. 2011;40(6):580-4. [CrossRef] [PubMed]

5. Hughes RL, Freilich RA, Bytell DE, Craig RM, Moran JM. Clinical conference in pulmonary disease. Aspiration and occult esophageal disorders. Chest. 1981;80(4):489-95. [CrossRef] [PubMed]

6. Tranovich VL, Buesching WJ, Becker WJ. Pathologic quiz case. Chronic pneumonia after gastrectomy. Pathologic diagnosis: chronic aspiration lipoid pneumonia with Mycobacterium abscessus. Arch Pathol Lab Med. 2001;125(7):976-8. [PubMed]

7. Jouannic I, Desrues B, Léna H, Quinquenel ML, Donnio PY, Delaval P. Exogenous lipoid pneumonia complicated by Mycobacterium fortuitum and Aspergillus fumigatus infections. Eur Respir J. 1996;9(1):172-4. [Pubmed]

8. Couto SS, Artacho CA. Mycobacterium fortuitum pneumonia in a cat and the role of lipid in the pathogenesis of atypical mycobacterial infections. Vet Pathol. 2007;44(4):543-6. [CrossRef] [PubMed]

9. Vadakekalam J, Ward MJ. Mycobacterium fortuitum lung abscess treated with ciprofloxacin. Thorax. 1991;46(10):737-8. [CrossRef] [PubMed] 